\title{
Organic piezoelectric materials: milestones and potential
}

\author{
Sarah Guerin', Syed A. M. Tofail ${ }^{1}$ and Damien Thompson ${ }^{1}$
}

\begin{abstract}
Research on the piezoelectric response of biomolecules has intensified following demonstration of open circuit voltages of over $20 \mathrm{~V}$ in biopiezoelectric generators. Organic piezoelectric nanotubes, fibers, and micro-islands have been grown and studied; however, the lack of fundamental understanding of the piezoelectric effect in nature hinders the rational design of biomaterials to provide a tailor-made piezoelectric response. Advances in high performance computing have facilitated the use of quantum mechanical calculations to predict the full piezoelectric tensor of biomolecular crystals, including amino acids and small peptides. By identifying directions of high piezoelectric response, the simulations can guide experimental crystal growth, device fabrication and electrical testing, which have led to the demonstration of unprecedented piezoelectric responses in organic crystals on the order of $200 \mathrm{pC} / \mathrm{N}$. These large responses arise from strong supramolecular dipoles, which can be tuned by molecular chemistry and packing, opening new opportunities for the realization of technologically useful piezoelectric devices from renewable materials. The amino acids predicted to exhibit the highest piezoelectric response, such as glycine, hydroxyproline and lysine, are anticipated to be used to engineer highly piezoelectric peptides in the future. With improved scaling of advanced computational methods, such as density functional perturbation theory, the research community can begin to efficiently screen peptide structures for enhanced electromechanical properties. This capability will accelerate the experimental development of devices and provide much-needed insight into the evolution of a hierarchical relation in biological materials starting from strongly piezoelectric building blocks.
\end{abstract}

Biological materials are low-symmetry, highly ordered structures that lack an inversion center. Thus, linear electromechanical coupling (piezoelectricity) is an inherent functional property of the majority of biomolecules. Bystrov et $\mathrm{al}^{1}$. describes biological piezoelectricity as being based on complex dipolar properties and dipole-dipole interactions mediated by intricate hydrogen bonding networks with different levels of self-assembly and hierarchy. In the past 60 years, piezoelectricity has been confirmed in a variety of biological materials, such as wood and bone, as well as fibrous proteins such as collagen, chitin, and elastin ${ }^{2}$, which present as highly ordered crystalline molecules in mammalian tissue. Classical piezoelectric principles have been applied to similar uniaxially orientated, bioactive polymers, such as poly

\footnotetext{
Correspondence: Damien Thompson (damien.thompson@ul.ie)

${ }^{1}$ Department of Physics, Bernal Institute, University of Limerick, Limerick V94 T9PX, Ireland
}

(L-lactic acid) (PLLA), poly ( $\gamma$-benzyl glutamate) (PBG), and cellulose ${ }^{3}$. These biodegradable polymers have been used as piezoelectric implants to enhance bone formation in both pure and compound forms ${ }^{4}$. Until the 21 st century, biological piezoelectricity was not considered technologically significant, with experimental values ranging from 0.1 to $2 \mathrm{pC} / \mathrm{N}^{2}$. For comparison, commercially exploited piezoelectrics, such as aluminum nitride (AlN) and lead zirconate titanate $(\mathrm{PZT})^{5}$, show piezoelectric constants of approximately $8 \mathrm{pC} / \mathrm{N}$ and $800 \mathrm{pC} / \mathrm{N}$, respectively. A comparison of the piezoelectric strain constants of biological materials to date with a variety of inorganic piezoelectrics is shown in Fig. 1. For further comparisons, readers are directed to the works of Aizen ${ }^{6}$, Shrout ${ }^{7}, \mathrm{Chorsi}^{8}$, and Wei ${ }^{9}$.

Spurred by demonstration of open circuit voltages of over $20 \mathrm{~V}$ in piezoelectric generators made of viruses ${ }^{10}$, prawn shells $^{11}$, and fish bladders ${ }^{12}$ (see examples, Fig. 1b), 


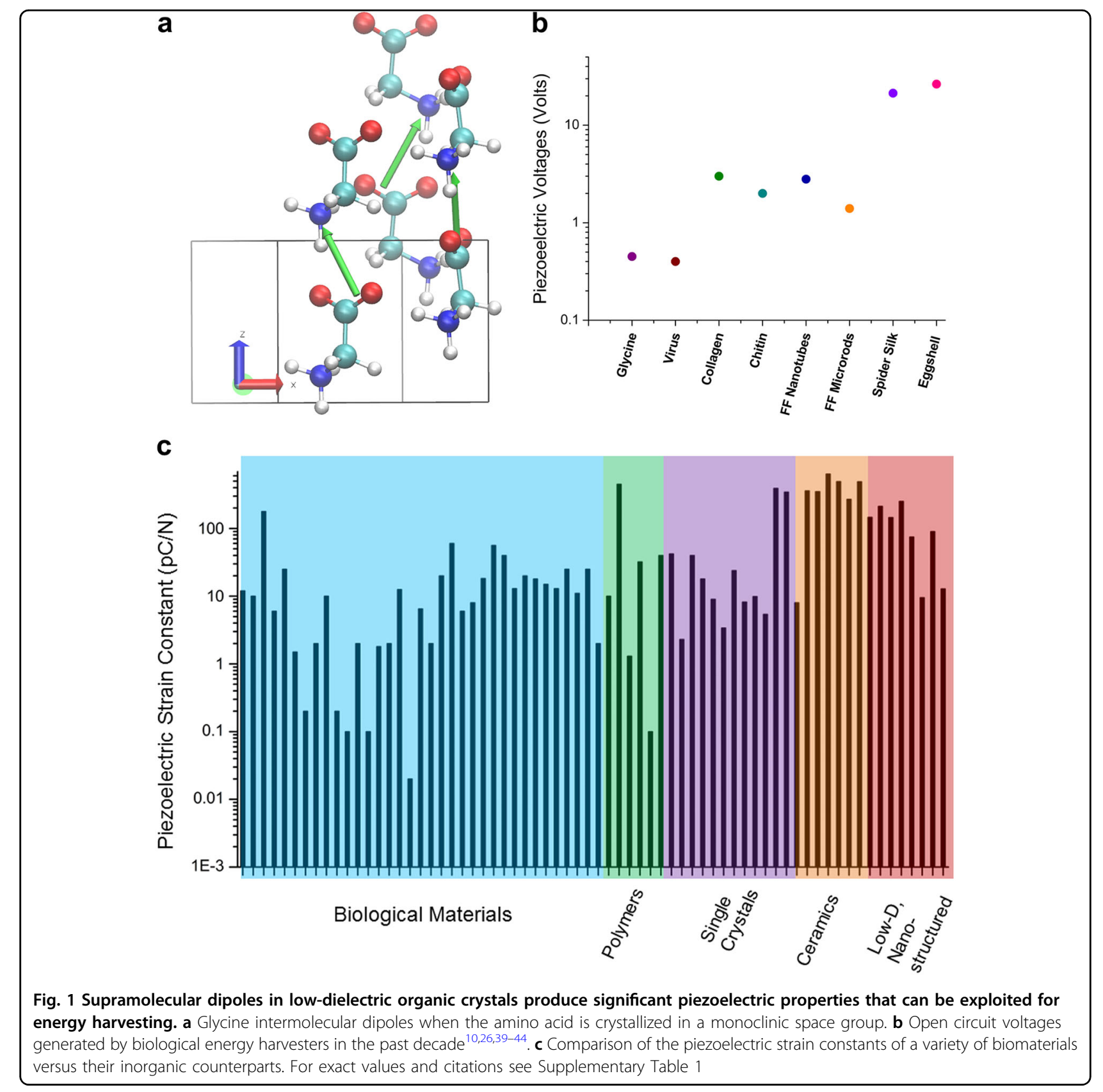

biological piezoelectric research has intensified in recent years. Organic piezoelectric nanotubes, fibers and microislands have been grown and studied ${ }^{13}$; however, the lack of fundamental understanding of the piezoelectric effect in biomolecules hinders the design of tailor-made piezoelectric responses in materials engineering. For inorganic piezoelectrics, atomic-scale investigations continue to highlight how a nanoscale understanding of piezoelectricity is necessary to enhance macroscopic electromechanical properties ${ }^{14-16}$.

Quartz, zinc oxide, and AlN are three classically exploited piezoelectric materials ${ }^{17}$. To study and quantify fundamental piezoelectric properties, researchers have historically focused on single crystals from which the behavior of the bulk material can be understood by the repeated pattern of atomic or molecular-scale unit cells. However, this approach has not been the case for biological piezoelectricity. The majority of experimental investigations into biological piezoelectricity in the past 60 years have been on macroscopic or polymeric samples. This can be attributed in part to the extreme difficulties in crystallizing fibrous and transmembrane proteins, but a large number of amino acids and peptides have been crystallized since the advent of X-ray diffraction ${ }^{18}$ and, in 

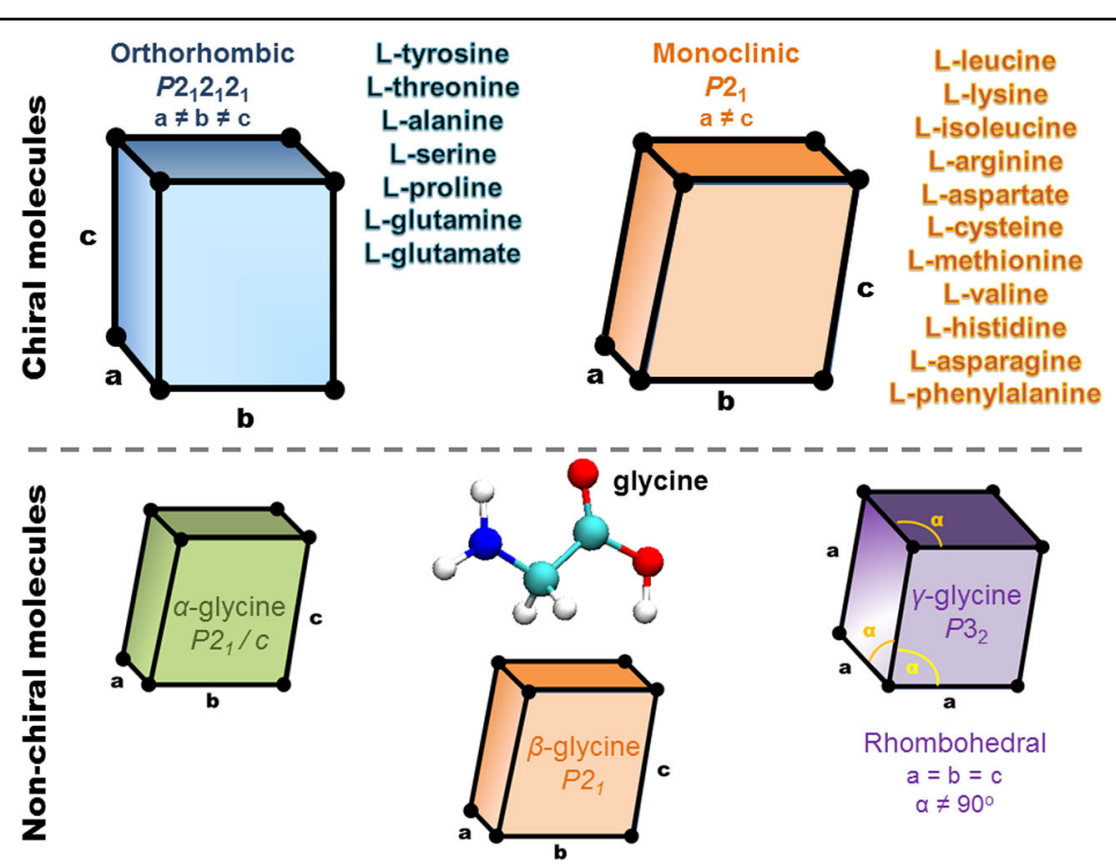

Rhombohedral

$a=b=c$ $a \neq 90^{\circ}$

Fig. 2 The crystal symmetries of the proteinogenic amino acid crystals

particular, studied for their nonlinear optical (NLO) properties $^{19}$. Lysozyme, a well-known globular protein found in tears and saliva, was only recently reported to exhibit piezoelectric properties in crystalline forms ${ }^{20}$. In addition to lysozyme, one of the nucleobases, thymine, was shown to be ferroelectric and hence piezoelectric in the crystalline form ${ }^{21}$. Biological materials present new challenges in the field of piezoelectricity, most notably predicting the role of water in modulating the measured dielectric constants and thus piezoelectric properties. A further bottleneck is the inherent softness of biological materials in a field where measurements have traditionally required application of external mechanical forces. The small size of the majority of biomolecular crystals also limits how electrical contact can be made-particularly compared to large inorganic piezoceramics that can be sliced, polished and electroded with relative ease.

Single crystal studies present a number of advantages for investigating piezoelectricity in any material, including biological structures. First, in terms of theoretical methodology, quantum mechanical modeling most often begins with the unit cell. Density functional theory $(\mathrm{DFT})^{22}$ utilizes periodic boundary conditions to simulate bulk material behavior and can quantify material physical properties of crystals, including the dielectric, elastic, and piezoelectric constants. By studying biomolecular crystals in this way, the predicted physical properties can be directly related to single crystal experiments, allowing effective screening of organic crystals for experimental investigation. A combination of modeling and characterization can provide much-needed insight into how piezoelectric properties are modulated by unit cell properties, such as dipole moments, molecular packing, and composition (Fig. 1a). Figure 1a shows that crystallizing biomolecules creates a network of unit cell dipoles identical to the mechanisms of classical inorganic piezoelectrics, which allows for biological single crystals to easily fulfill the role of piezoceramics, e.g., in stack actuation. Single crystals can often be grown quite easily from aqueous solutions, and the quality and shape of organic single crystals can be modulated using additives or $\mathrm{pH}$ buffers. This simple idea that the properties of single crystals can be modified chemically to enhance their properties suggests that this could be extended to piezoelectric properties using theoretical predictions to guide experiments.

Lemanov first suggested in $2000^{23}$ that the key to understanding biological piezoelectricity was to study the 20 protein amino acids that are used to build all biological materials. Amino acids crystallize primarily in lowsymmetry orthorhombic and monoclinic space groups (Fig. 2). These space groups are noncentrosymmetric, which lend themselves to piezoelectric and NLO properties, with the exception of the largest amino acid, Ltryptophan, and glycine. L-tryptophan crystallizes with the lowest $P 1$ crystal symmetry ${ }^{24}$. Glycine is not chiral thus it does not have an enantiomorphic mirror image, and it crystallizes in three distinct crystal symmetries: $\alpha, \beta$, and $\gamma^{25}$. The only amino acid structure that precludes piezoelectricity is $\alpha$-glycine. The highest responses 


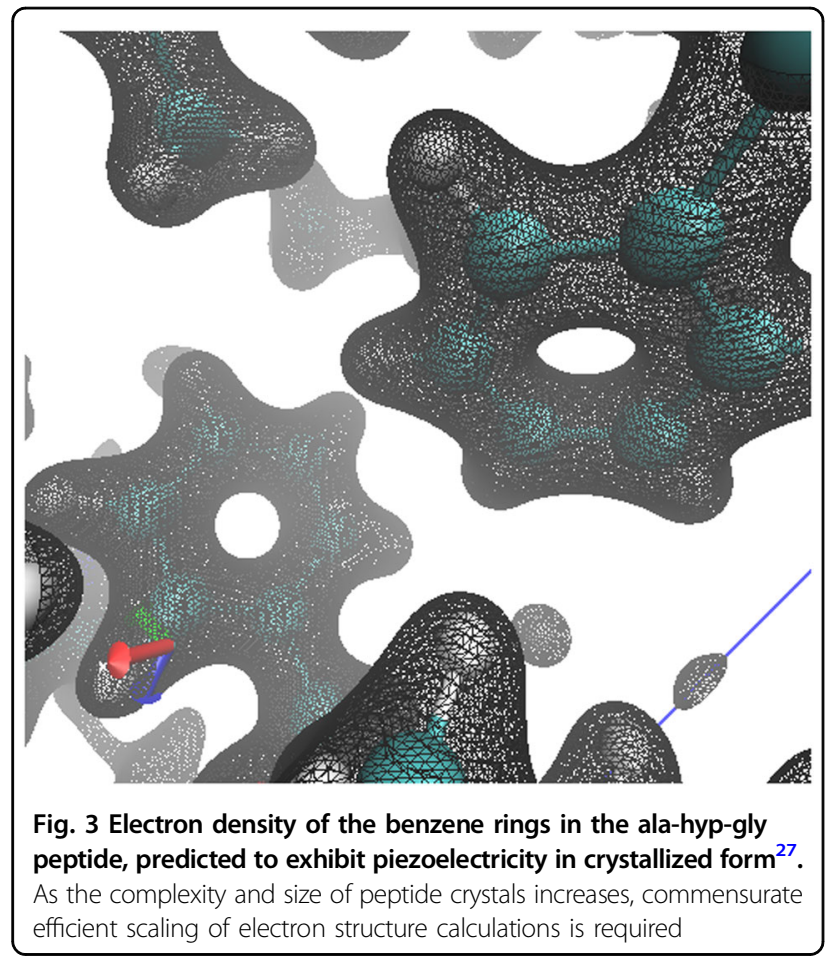

measured so far in amino acid single crystals are $178 \mathrm{pC} /$ $\mathrm{N}$ and $25 \mathrm{pC} / \mathrm{N}$ in $\beta$-glycine and hydroxy-L-proline, respectively ${ }^{26,27}$.

In 2012, Lemanov ${ }^{28}$ fired high frequency electrical pulses at crystalline amino acid powders (not single crystals) and found that DL-alanine and $\gamma$-glycine had the strongest piezoelectric response of the amino acids studied. This result is consistent with recent quantitative investigations into longitudinal piezoelectricity in these crystals, ${ }^{26,29}$ which determined that both DL-alanine and $\gamma$-glycine crystallize in unique space groups that allow for a longitudinal $d_{33}$ constant of $10 \mathrm{pC} / \mathrm{N}$. Lemanov did not detect piezoelectricity in L-cysteine or L-isoleucine powders, which are noncentrosymmetric in their single crystal form. This result highlights the importance of using crystallized amino acids as opposed to powders when studying chiral molecules. A false piezoelectric signal was also measured in $\alpha$-glycine, which was attributed to contamination from other noncentrosymmetric polymorphs. Furthermore, an orientation dependence of the piezoelectric response of glycine powder was noted, which has since been explained quantitatively ${ }^{26}$. When $\gamma$-glycine crystals are rotated sequentially by $90^{\circ}$ between electrodes (so that their crystallographic a, b, and c axes become perpendicular to the plane of electrical contacts), their measured piezoelectric constant changes from approximately 1 to 2 , and finally to $10 \mathrm{pC} / \mathrm{N}^{26}$. Prior to Lemanov's work in 2000, the only experimental investigation into amino acid piezoelectricity had been performed by Vasilescu et al. in $1970 .^{30}$ The noncentrosymmetry of the amino acids was confirmed in 1967 by NLO methods ${ }^{31}$.
Amino acid compound crystals have been more widely studied for their piezoelectric properties, including $\mathrm{L}$-arginine phosphate $(\mathrm{LAP})^{32}$ and triglycine sulfate (TGS $^{33}$. Doping of centrosymmetric $\alpha$-glycine crystals with other L-amino acids also leads to crystals with piezoelectric properties $^{34}$. The peptide that has attracted the most attention for its piezoelectric properties is di-phenylalanine (FF), which is easily influenced by external electric fields in aqueous solution $^{35}$. FF peptide nanotubes have been confirmed to be piezoelectric and pyroelectric ${ }^{1,36,37}$. These peptides also demonstrate high stability and a large Young's Modulus, which has made them potential alternatives to a number of piezoelectrics widely used in consumer electronics. Recent work has shown that FF nanogenerators can generate voltages of $0.6-2.8 \mathrm{~V}$, which places them alongside glycine and alanine as ideal candidates for self-powered brain implants and pacemakers ${ }^{38}$.

New challenges arise for predictive modeling as we continue up the biomolecular hierarchy, specifically to peptide and protein crystals (Fig. 3). Although trends in piezoelectric charge tensors can be seen in the sequences of small peptide crystals ${ }^{27}$, it is unrealistic to track, for example, elastic constants through the hierarchy of biomolecular crystals. The amino acids predicted to exhibit the highest piezoelectric response, such as glycine and lysine, are thus anticipated to be used to engineer highly piezoelectric peptides in the future. With improved scaling of advanced quantum mechanical methods, such as density functional perturbation theory (DFPT, peptide structures can be efficiently screened for enhanced electromechanical properties.

To conclude, piezoelectricity is a ubiquitous yet underexploited property of the majority of biomolecules. Studying biomolecules in their crystallized form allows for the understanding of their electromechanical behavior in terms of unit cell properties. A single crystal approach is needed to fully understand and exploit biological piezoelectricity, allowing for high-throughput, simulationguided experiments. A solid-state physics perspective on biomaterials will aid the adaptation of biological crystals in high performance sensors, actuators, and energy harvesters. Biological piezoelectricity also holds unique potential for in vivo sensing and drug delivery, which is diversifying current research beyond the replacement of lead-based piezo materials and requires bold advances in predictive modeling of the electronic structures of large biomolecular crystals and supramolecular assemblies.

Conflict of interest

The authors declare that they have no conflict of interest.

\section{Publisher's note}

Springer Nature remains neutral with regard to jurisdictional claims in published maps and institutional affiliations. 
Supplementary information is available for this paper at https://doi.org/ 10.1038/s41427-019-0110-5.

Received: 9 July 2018 Revised: 14 November 2018 Accepted: 11 December 2018.

Published online: 7 March 2019

\section{References}

1. Bystrov, V., Seyedhosseini, E., Kopyl, S., Bdikin, I. \& Kholkin, A. Piezoelectricity and ferroelectricity in biomaterials: molecular modeling and piezoresponse force microscopy measurements. J. Appl. Phys. 116, 066803 (2014).

2. Fukada, E. History and recent progress in piezoelectric polymers. Ultrason., Ferroelectr., Freq. Control, IEEE Trans. 47, 1277-1290 (2000).

3. Fukada, E. Bioelectrets and biopiezoelectricity. IEEE Trans. Electr. Insul. 27, 813-819 (1992).

4. Shikinami, Y. \& Okuno, M. Bioresorbable devices made of forged composites of hydroxyapatite (HA) particles and poly L-lactide (PLLA). Part II: practical properties of miniscrews and miniplates. Biomaterials 22, 3197-3211 (2001).

5. Lobl, H. et al. in Ultrasonics Symposium, 1999. Proceedings. 1999 IEEE. 1031-1036 (IEEE).

6. Aizen, R., Tao, K., Rencus-Lazar, S. \& Gazit, E. Functional metabolite assemblies - a review. J. Nanopart. Res. 20, 125 (2018).

7. Shrout, T. R. \& Zhang, S. J. Lead-free piezoelectric ceramics: alternatives for PZT? J. Electroceram. 19, 113-126 (2007).

8. Chorsi, M. T. et al. Piezoelectric biomaterials for sensors and actuators. Adv. Mater. 1802084 (2018).

9. Wei, $\mathrm{H}$. et al. An overview of lead-free piezoelectric materials and devices. J. Mater. Chem. C 6, 12446-12467 (2018).

10. Lee, B. Y. et al. Virus-based piezoelectric energy generation. Nat. Nanotechnol. 7, 351-356 (2012).

11. Ghosh, S. K. \& Mandal, D. Bio-assembled, piezoelectric prawn shell made selfpowered wearable sensor for non-invasive physiological signal monitoring. Appl. Phys. Lett. 110, 123701 (2017)

12. Ghosh, S. K. \& Mandal, D. Efficient natural piezoelectric nanogenerator: electricity generation from fish swim bladder. Nano Energy 28, 356-365 (2016)

13. Seyedhosseini, E. et al. Self-assembly of organic ferroelectrics by evaporative dewetting: a case of $\beta$-glycine. ACS Appl. Mater. \& Interfaces 9, 20029-20037 (2017).

14. Ico, G., Myung, A., Kim, B. S., Myung, N. V. \& Nam, J. Transformative piezoelectric enhancement of P (VDF-TrFE) synergistically driven by nanoscale dimensional reduction and thermal treatment. Nanoscale 10, 2894-2901 (2017).

15. Wang, Z. L. \& Song, J. Piezoelectric nanogenerators based on zinc oxide nanowire arrays. Science 312, 242-246 (2006).

16. Song, $X$. et al. Enhanced piezoelectric effect at the edges of stepped molybdenum disulfide nanosheets. Nanoscale 9, 6237-6245 (2017).

17. Collins, J., Hagon, P. \& Pulliam, G. Evaluation of new single crystal piezoelectric materials for surface acoustic-wave applications. Ultrasonics 8, 218-226 (1970).

18. Williams, P. A., Hughes, C. E. \& Harris, K. D. L-Lysine: exploiting powder X-ray diffraction to complete the set of crystal structures of the 20 directly encoded proteinogenic amino acids. Angew. Chem. Int. Ed. 54, 3973-3977 (2015).

19. Moitra, S. \& Kar, T. Growth and characterization of L-valine-a nonlinear optical crystal. Cryst. Res. Technol. 45, 70-74 (2010).

20. Stapleton, A. et al. The direct piezoelectric effect in the globular protein lysozyme. Appl. Phys. Lett. 111, 142902 (2017).
21. Bdikin, I. et al. Local piezoresponse and polarization switching in nucleobase thymine microcrystals. J. Appl. Phys. 118, 072007 (2015).

22. Jones, R. O. Density functional theory: Its origins, rise to prominence, and future. Rev. Mod. Phys. 87, 897-923 (2015).

23. Lemanov, $V$. Piezoelectric and pyroelectric properties of protein amino acids as basic materials of soft state physics. Ferroelectrics $\mathbf{2 3 8}, \mathbf{2 1 1 - 2 1 8}$ (2000).

24. Görbitz, C. H., Törnroos, K. W. \& Day, G. M. Single-crystal investigation of Itryptophan with $Z^{\prime}=16$. Acta Crystallogr. B 68, 549-557 (2012).

25. Boldyreva, E. et al. Polymorphism of glycine, Part I. J. Therm. Anal. Calorim. 73 409-418 (2003).

26. Guerin, S. et al. Control of piezoelectricity in amino acids by supramolecular packing. Nat. Mater. 17, 180 (2017).

27. Guerin, S., Syed, T. A. \& Thompson, D. Deconstructing collagen piezoelectricity using alanine-hydroxyproline-glycine building blocks. Nanoscale 10, 9653-9663 (2018)

28. Lemanov, V. Ferroelectric and piezoelectric properties of protein amino acids and their compounds. Phys. Solid State 54, 1841-1842 (2012).

29. Guerin, S et al. Racemic amino acid piezoelectric transducer. Phys. Rev. Lett accepted (2019).

30. Vasilescu, D., Cornillon, R. \& Mallet, G. Piezoelectric resonances in amino-acids Nature 225, 635 (1970).

31. Delfino, M. A comprehensive optical second harmonic generation study of the non-centrosymmetric character of biological structures. Mol. Cryst. Liq. Cryst. 52, 271-284 (1979).

32. Sil'vestrova, I. et al. Elastic, piezoelectric, and dielectric properties of LAP crystals. Sov. Phys. Crystallogr. 37, 831-834 (1992).

33. Ikeda, T., Tanaka, Y. \& Toyoda, H. Piezoelectric properties of triglycine-sulphate. Jpn. J. Appl. Phys. 1, 13 (1962).

34. Meirzadeh, E. et al. Origin and structure of polar domains in doped molecular crystals. Nat. Commun. 7, 13351 (2016).

35. Kelly, C. M. et al. Conformational dynamics and aggregation behavior of piezoelectric diphenylalanine peptides in an external electric field. Biophys. Chem. 196, 16-24 (2015).

36. Kholkin, A., Amdursky, N., Bdikin, I., Gazit, E. \& Rosenman, G. Strong piezoelectricity in bioinspired peptide nanotubes. ACS nano 4, 610-614 (2010).

37. Esin, A. et al. Pyroelectric effect and polarization instability in self-assembled diphenylalanine microtubes. Appl. Phys. Lett. 109, 142902 (2016).

38. Jenkins, K., Kelly, S., Nguyen, V., Wu, Y. \& Yang, R. Piezoelectric diphenylalanine peptide for greatly improved flexible nanogenerators. Nano Energy, 51, 317-323 (2018).

39. Karan, S. K. et al. A new insight towards eggshell membrane as high energy conversion efficient bio-piezoelectric energy harvester. Mater. Today Energy $\mathbf{9}$, 114-125 (2018).

40. Karan, S. K. et al. Nature driven spider silk as high energy conversion efficient bio-piezoelectric nanogenerator. Nano Energy 49, 655-666 (2018).

41. Nguyen, V., Zhu, R., Jenkins, K. \& Yang, R. Self-assembly of diphenylalanine peptide with controlled polarization for power generation. Nat. Commun. 7, 13566 (2016).

42. Ghosh, S. K. \& Mandal, D. High-performance bio-piezoelectric nanogenerator made with fish scale. Appl. Phys. Lett. 109, 103701 (2016).

43. Hoque, N. A. et al. Biowaste crab shell-extracted chitin nanofiber-based superior piezoelectric nanogenerator. J. Mater. Chem. A 6, 13848-13858 (2018).

44. Lee, J.-H. et al. Diphenylalanine peptide nanotube energy harvesters. ACS nano 12, 8138-8144 (2018). 